\title{
Matéria e nutrientes da parte aérea de adubos verdes em cultivos exclusivo e consorciado ${ }^{1}$
}

\author{
Graciela Bassan Rodrigues², Marco Eustáquio de Sá3, Walter Veriano Valério Filho4 , Salatier Buzetti ${ }^{5}$, \\ Danila Comelis Bertolin ${ }^{2 *}$, Ticiana Petean Pina ${ }^{6}$
}

\begin{abstract}
RESUMO
O objetivo deste estudo foi verificar os efeitos de diferentes coberturas do solo utilizando leguminosas (guandu, crotalária juncea, feijão-de-porco e mucuna-preta) e uma gramínea (milheto) isolada e em consórcio leguminosa/gramínea e uma área de pousio, sobre a produção de matéria, nos teores e no acúmulo de nutrientes. O delineamento experimental adotado foi o de blocos casualizados, com 10 tratamentos (guandu, crotalária, feijão-de-porco, mucuna-preta, milheto, guandu + milheto, crotalária + miheto, feijão-de-porco + milheto, mucuna-preta + milheto e uma área de pousio) e oito repetições. O experimento foi conduzido, no ano agrícola 2006/07, na área experimental da UNESP - Campus de Ilha Solteira, localizada no município de Selvíria-MS, em solo anteriormente ocupado por vegetação de cerrado. A produção de matéria fresca e seca das plantas e os teores e acúmulo de nutrientes das plantas de cobertura foram obtidos por meio da coleta de oito amostras aleatórias de cada planta . Verificou-se que ocorreram diferenças significativas entre as espécies tanto isoladamente como em consórcio em cada unidade experimental. A combinação que proporcionou maiores resultados para a matéria seca foi mucuna + milheto, seguida de crotalária + milheto com 23,3 e 14,8 $\mathrm{t} \mathrm{ha}^{-1}$ respectivamente. Para acúmulo de nitrogênio foram obtidos os valores máximos de 35,1 e 31,0 $\mathrm{g} \mathrm{kg}^{-1} \mathrm{para}$ mucuna e o feijão-de-porco e para fósforo 3,0; 2,7 e 2,5 $\mathrm{g} \mathrm{kg}^{-1}$ para feijão-de-porco, guandu e mucuna, evidenciando o potencial das culturas consorciadas com milheto e da mucuna, feijão-de-porco e guandu para utilização como espécies de cobertura.
\end{abstract}

Palavras-chave: Pennisetum glaucum (L.) R. Brown, , plantas de cobertura, plantio direto, leguminosas, reciclagem de nutrientes.

\section{ABSTRACT}

\section{Shoot biomass and nutrients of green manure in monoculture and intercropped systems}

The objective of this work was to verify the effects of different cover crops using legumes (pigeon pea, sunn hemp, jack bean and velvet bean) in monoculture and intercropped systems of grass (pearl millet), legumes/grass and a fallow area, on phytomass production and nutrient return. The experimental design was randomized blocks with ten treatments (pigeon pea, sunn hemp, jack bean, velvet bean, pearl millet, pigeon pea + pearl millet, sunn hemp + pearl millet, jack bean + pearl millet, velvet bean + pearl millet one more fallow area) and eight repetitions. The experiment was conducted

Recebido para publicação em 08/02/2010 e aprovado em 03/05/2012

${ }^{1}$ Parte da Dissertação de Mestrado da primeira autora, bolsista da Fundação de Amparo ao Ensino e Pesquisa de São Paulo (FAPESP).

${ }^{2}$ Engenheira-Agrônoma, Doutoranda. Departamento de Fitotecnia, Tecnologia de Alimentos e Sócio-Economia, Faculdade de Engenharia de Ilha Solteira (FEIS/UNESP), Avenida Brasil, Centro, 56, 15385-000, Ilha Solteira, São Paulo, Brasil. gra_bassan@yahoo.com.br; danilacomelis@hotmail.com (*Autora correspondente)

${ }^{3}$ Engenheiro-Agrônomo, Doutor. Departamento de Fitotecnia, Tecnologia de Alimentos e Sócio-Economia, Faculdade de Engenharia de Ilha Solteira (FEIS/UNESP), Avenida Brasil, Centro, 56, 15385-000, Ilha Solteira, São Paulo, Brasil. mesa@agr.feis.unesp.br

${ }^{4}$ Estatístico, Doutor. Departamento de Matemática, Faculdade de Engenharia de Ilha Solteira (FEIS/UNESP). Avenida Brasil, Centro, 56, 15385-000, Ilha Solteira, São Paulo, Brasil. wvvf@mat.feis.unesp.br

${ }^{5}$ Engenheiro-Agrônomo, Doutor. Departamento de Fitossanidade, Engenharia Rural e Solos, Faculdade de Engenharia de Ilha Solteira (FEIS/UNESP). Avenida Brasil, Centro, 56, 15385-000, Ilha Solteira, São Paulo, Brasil.sbuzetti@agr.feis.unesp.br

${ }^{6}$ Engenheira-Agrônoma, Mestranda. Departamento de Fitotecnia, Tecnologia de Alimentos e Sócio-Economia, Faculdade de Engenharia de Ilha Solteira (FEIS/UNESP), Avenida Brasil, Centro, 56, 15385-000, Illha Solteira, São Paulo, Brasil. tici_agro@hotmail.com 
in the agricultural year 2006/07, in the experimental area of UNESP-Campus de Ilha Solteira, Selvíria, MS, in soil previously occupied with cerrado vegetation. The production of plant green and dry mass, return of nutrients of the cover crops were calculated through the collection of 8 random samples of each cover crop. It was found significant differences among the crops both in monoculture and in intercropped system for each experimental unit. The combination that provided in the highest dry mass and nutrient return was velvet bean + pearl millet, followed by sunn hemp + pearl millet.

Key words: Pennisetum glaucum (L.) R. Brown, cover crops, no till, legumes, recycling of nutrients.

\section{INTRODUÇÃO}

No sistema plantio direto, a cobertura morta na superfície do solo é o principal componente do sucesso, atuando como reguladora de temperatura e da água do solo, no enriquecimento de matéria orgânica, como barreira física a algumas plantas daninhas, na prevenção das diversas modalidades de erosão, entre outros (Braz et al., 2010).

O emprego de leguminosas como cultura de cobertura se destaca, pois além de proporcionarem benefícios semelhantes aos de outras espécies, apresentam a capacidade de acumular $\mathrm{N}$ pela fixação biológica (Braz et al., 2010; Silva et al., 2009).

Já com o uso de gramíneas, considerando uma relação C/N mais alta, a sua taxa de decomposição é mais lenta do que as leguminosas, o que permite a manutenção de palha na superfície do solo por maior espaço de tempo. Por outro lado, o aporte de nutrientes e a taxa de mineralização mais rápida das leguminosas tornam a palhada dessas plantas mais atrativa para a composição de um sistema de rotação de culturas, porém é importante considerar que em condições tropicais é interessante manter ou aumentar os níveis de matéria orgânica do solo. Assim, parece viável que a associação gramínea/leguminosa possa favorecer esses objetivos, e dessa forma estudos que congreguem essa junção são bastante relevantes.

Segundo Pitol et al. (2006), na agricultura tradicional os produtores têm se deparado com uma série de limitações e dificuldades quanto ao uso de adubos verdes, as quais poderão ser superadas à medida que, no sistema de produção, se utilizarem de práticas de manejo como plantio direto, rotação de culturas, consórcios e integração lavoura-pecuária.

Os adubos verdes são de grande importância para a agricultura, pois promovem a ciclagem mais rápida de nutrientes, favorecendo seu uso pela cultura em sequência, principalmente daqueles elementos com potencial de lixiviação como o nitrogênio e os cátions trocáveis ou dos que podem ser retidos com relativa facilidade, como o fósforo em solos intemperizados. O guandu, por exemplo, pode promover a liberação do fósforo comumente adsorvido aos óxidos e hidróxidos de Fe nos solos (Ae et al., 1990). No entanto, essa utilização eficiente dos nutrientes liberados no processo de decomposição depende do sistema de cultivo (rotação, sucessão ou consórcio) e da potencial combinação em termos de maximização de resultados entre a cultura principal e os adubos verdes (Pitol et al., 2006).

De acordo com Bordin et al. (2008) o guandu e o milheto apresentam alto potencial de produção de matéria seca quando submetidos à escarificação podendo o milheto prover até $15,3 \mathrm{t} \mathrm{ha}^{-1}$ e o guandu $12,29 \mathrm{t} \mathrm{ha}^{-1}$. Resultados obtidos por Heinrichs et al. (2005) em estudo sobre rendimento de matéria fresca de mucuna anã, guandu anão, crotalária e feijão-de-porco em cultivo consorciado com o milho destacam as características do feijão-de-porco com superioridade de produção de matéria fresca da ordem de 290 a 82\%. Para Ferrari Neto et al. (2011) o guandu e o milheto em cultivos solteiros e o consórcio milheto + guandu podem proporcionar incremento nos parâmetros produtivos do mamoeiro em sucessão de cultivos com produção de matéria seca de 4,7; 14,0 e 6,2 t ha ${ }^{-1}$.

Há uma grande quantidade de espécies promissoras para utilização como plantas de cobertura, e, sendo assim, o objetivo deste trabalho foi verificar os efeitos de diferentes coberturas do solo utilizando leguminosas (guandu, crotalária juncea, feijão-de-porco e mucuna-preta) e uma gramínea (milheto) em cultivo isolado e em consórcio leguminosa/gramínea sobre a produção de matéria e acúmulos de nutrientes da parte aérea das plantas.

\section{MATERIAL E MÉTODOS}

O experimento foi conduzido no município de Selvíria, Estado do Mato Grosso do Sul, na Fazenda de Ensino e Pesquisa da Unesp, Campus de Ilha Solteira. A área está situada aproximadamente a $51^{\circ} 22^{\prime}$ O e e $20^{\circ} 22^{\prime}$ ' S, com altitude de 335 metros. O clima é do tipo Aw, segundo a clas- 
sificação de Köeppen, apresentando temperatura média anual de $23,5^{\circ} \mathrm{C}$, precipitação pluvial anual média de 1.370 mm e a umidade relativa do ar oscila entre 70 e $80 \%$. O solo do local, que no passado era ocupado por vegetação de Cerrado, foi classificado como Latossolo Vermelho Distrófico típico argiloso, A moderado, hipodistrófico álico, caulinítico, férrico, compactado, muito profundo, moderadamente ácido (Embrapa, 1999).

As características químicas do solo foram determinadas antes da instalação do experimento, seguindo a metodologia proposta por Raij \& Quaggio (1983), e os resultados são apresentados a seguir: matéria orgânica 28,0 $\mathrm{g} \mathrm{dm}^{-3} ; \mathrm{pH}\left(\mathrm{CaCl}_{2}\right)$ 5,1; P (resina) $5,0 \mathrm{mg} \mathrm{dm}^{-3} ; \mathrm{K}, \mathrm{Ca}, \mathrm{Mg}, \mathrm{H}$ $+\mathrm{Al}$, SB e CTC 1,4; 22,0; 10,0; 22,0; 33,0; e 54,0 $\mathrm{mmol}_{\mathrm{c}} \mathrm{dm}^{-3}$, respectivamente, e saturação por bases de $60 \%$.

Para a implantação das culturas de cobertura no verão, o preparo do solo foi realizado por meio de uma aração com arado de aiveca e duas gradagens. A semeadura das plantas de cobertura foi realizada manualmente em novembro de 2006, sendo utilizado o delineamento em blocos casualizados com 10 tratamentos e 8 repetições.

Os tratamentos foram: guandu (Cajanus cajan), crotalária juncea (Crotalaria juncea), feijão-de-porco (Canavalia ensiformis), mucuna-preta (Stizolobium aterrimum), milheto (Pennisetum glaucum); os consórcios: guandu + milheto, crotalária + miheto, feijão-de-porco + milheto, mucuna-preta + milheto e uma área de pousio. As combinações das leguminosas com o milheto foram na proporção de $50 \%$ da população de cada uma, em espaçamento de $0,50 \mathrm{~m}$ entrelinhas.

Cada parcela constou de 10 linhas de $10 \mathrm{~m}$. O número de sementes/m para cada uma das plantas de coberturas foi: milheto (80 sementes/m), mucuna (10 sementes $/ \mathrm{m}$ ), guandu (20 sementes/m), feijão-de-porco (12 sementes/ m) e crotalária (30 sementes/m), de acordo com as recomendações de Ambrosano e Wutke (1997).

A produção de matéria fresca e seca das plantas de cobertura foi obtida pela coleta de oito amostras aleatórias de $0,25 \mathrm{~m}^{2}$ de cada planta de cobertura aos 120 dias após semeadura, em que as plantas foram cortadas rente ao solo, pesadas e determinada a produção de matéria fresca, sendo os dados transformados em t/ha de matéria fresca. Posteriormente, foi realizada a lavagem e feito o acondicionamento em sacos de papel e secagem em estufa a $65^{\circ} \mathrm{C}$. Após seco, o material foi pesado e os resultados obtidos foram transformados em $\mathrm{t} /$ ha de matéria seca.

Determinaram-se os teores de N, P, K, Ca, Mg, S, B, Fe, Mn e Zn. Para determinação desses teores, as amostras foram moídas em moinho tipo Wiley e foram utilizadas as amostras das oito repetições coletadas no campo de cada espécie. Para a determinação do teor de N, foi realizada digestão sulfúrica segundo o método semimicro Kjeldahl, para a determinação do teor de B foi realizada digestão por via seca e para os demais nutrientes foi realizada a digestão nitroperclórica, sendo os teores de $\mathrm{P}$ e o $\mathrm{B}$ determinados por colorimetria, e o de $\mathrm{S}$ por turbidimetria. Os teores de $\mathrm{K}, \mathrm{Ca}, \mathrm{Mg}$, Mn e $\mathrm{Zn}$ foram determinados por espectrofotometria de absorção atômica. Os teores de macro e micronutrientes foram expressos em $\mathrm{g} \mathrm{kg}^{-1} \mathrm{de}$ MS e $\mathrm{mg} \mathrm{kg}^{-1}$ de MS, respectivamente. O acúmulo de macronutrientes foi obtido pelo produto da quantidade de matéria seca com o teor dos nutrientes da parte aérea das culturas de cobertura.

Foi realizada análise de variância para verificar se houveram diferenças entre os tratamentos, havendo diferenças foi realizado o teste de Scott-Knott a 5\% de probabilidade (Banzatto e Kronka, 2006). O programa estatístico utilizado foi o Sanest (Zonta e Machado, 1991).

\section{RESULTADOS E DISCUSSÕES}

Na Figura 1, encontram-se os dados referentes à temperatura média, precipitação total e umidade relativa do ar durante o período de condução do experimento os quais foram favoráveis ao desenvolvimento das culturas de cobertura.

Para matéria fresca e seca das plantas de cobertura (Tabela 1), verifica-se que a combinação mucuna + milheto proporcionou as maiores produções, diferindo significativamente dos demais tratamentos. O consórcio gramínea/ leguminosa resultou em maiores quantidades de matéria fresca quando comparadas ao cultivo isolado, exceção apenas para o milheto quando cultivado isolado, concordando com os dados obtidos por Oliveira et al. (2002), que também utilizaram plantas de cobertura em cultivos isolado e consorciados em condições edafoclimáticas próximas.

A quantidade de matéria vegetal fornecida pelas plantas de cobertura, com exceção da mucuna-preta, esteve acima do que tem sido apontado como quantidade mínima ideal de adição de matéria seca em um sistema de rotação de culturas, de maneira que se mantenha adequada a cobertura do solo, que é de $6 \mathrm{t} \mathrm{ha}^{-1}$ ano ${ }^{-1}$ de palhada, conforme citado por Alvarenga et al., (2001), sendo a média geral de produtividade de matéria seca de $11,2 \mathrm{t} \mathrm{ha-1}$, valor quase que duas vezes a quantidade mínima, indicando assim boa cobertura do solo.

Quando cultivados solteiros, o milheto e a crotalária produziram 12,6 e 12 t ha ${ }^{-1}$ de matéria seca. Os valores observados estão de acordo com informações da literatura, que relatam acúmulos de matéria seca variando entre 5,02 e 6,15 t ha ${ }^{-1}$ na região de Cerrado de Goiás, segundo Amabile (1993); para o guandu; mais de $7 \mathrm{t} \mathrm{ha}^{-1} \mathrm{em}$ Mato Grosso do Sul, segundo Salton et al. (1993); e 55,2 $\mathrm{t} \mathrm{ha}^{-1}$ de matéria fresca (Bertolin et al. 2008); para a Crotalaria juncea, oscilando entre 5,7 e 19,8 tha $^{-1}$ em áreas de Cerrado do Distrito Federal, segundo Pereira (1991) para o fei- 


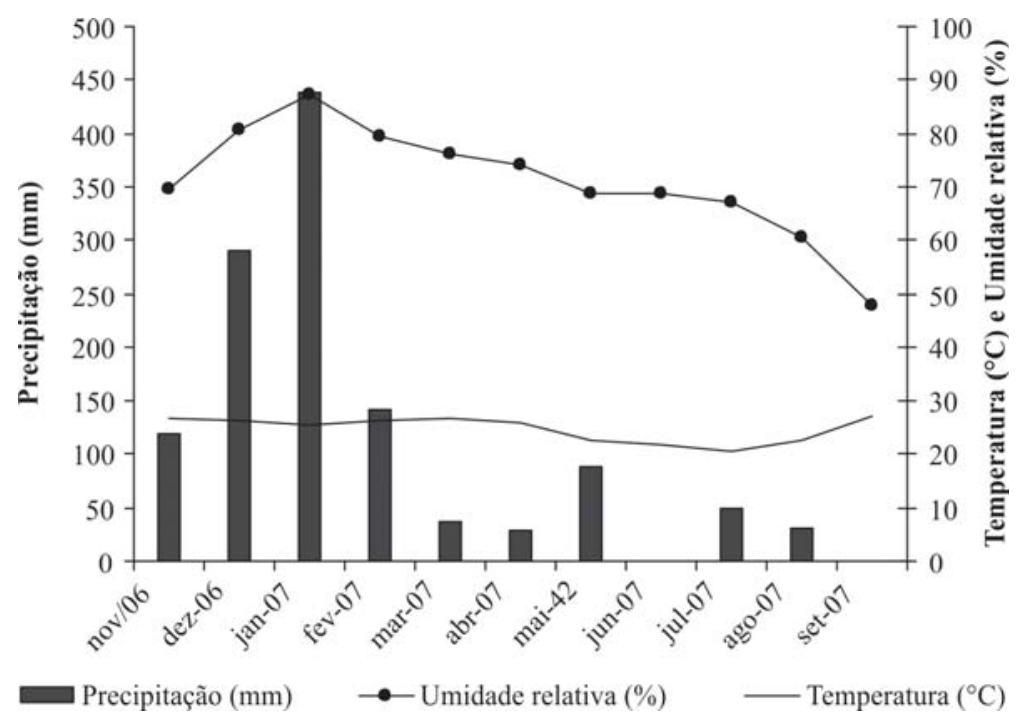

Figura 1. Precipitação pluviométrica, temperaturas médias e umidade relativa observadas nos meses de novembro de 2006 a março de 2007, em Selvíria-MS.

jão-de-porco; 4,8 t ha-1 ${ }^{-1}$ na região de Cerrado de Goiás, segundo Amabile et al. (2000) para a mucuna-preta e variando de 2,03 a 8,1 $\mathrm{t} \mathrm{ha}^{-1}$ no Cerrado do Brasil Central, segundo Spehar (1999) para o milheto.

De maneira geral, a produtividade de matérias verde e seca das leguminosas cultivadas solteiras (Tabela 1) encontra-se dentro dos valores obtidos por Teodoro et al. (2011), exceto para a mucuna-preta.

Teodoro et al. (2011) realizaram estudo com as leguminosas guandu, mucuna preta, Crotalaria spectabilis, crotalária juncea, mucuna cinza, lab-lab e feijão-de-porco e obtiveram valores de matéria seca entre 13,9 e 2,62 t ha $^{-1}$.

Quanto aos teores de nutrientes (Tabela 2), verificase que ocorreram diferenças significativas entre espécies e combinações de consórcio. Observa-se que quanto ao teor de $\mathrm{N}$ destacou-se a mucuna, vindo a seguir o feijãode-porco. Entre as combinações utilizadas, destaca-se a milheto + mucuna, que apresentou teor elevado de $\mathrm{N}$ de $\left(20,3 \mathrm{~g} \mathrm{~kg}^{-1}\right)$, mesmo tendo-se as matérias de uma gramínea e uma leguminosa.

De forma geral foi observado maior acúmulo de $\mathrm{N}>\mathrm{Ca}>\mathrm{K}>\mathrm{P}>\mathrm{Mg}>\mathrm{S}$, com exceção apenas do cultivo de milheto solteiro e do cultivo de milheto + guandu, onde o maior acúmulo observado foi de $\mathrm{N}>\mathrm{K}>\mathrm{Ca}>\mathrm{P}>\mathrm{Mg}>\mathrm{S}$. Resultado diferente foi obtido por Bordin et al. (2008) para feijão-de-porco e por Ferrari Neto et al. (2011) para guandu com maior acúmulo de potássio em relação ao cálcio.

Em condições ambientais similares, Oliveira (2002), trabalhando com sucessão de culturas, dentre elas guandu e milheto, observou que os teores de macronutrientes apresentaram baixo valor de variação entre as espécies, principalmente para nitrogênio, 25,07 e 10,13 $\mathrm{g} \mathrm{kg}^{-1}$, e fósforo, 3,14 e $1,43 \mathrm{~g} \mathrm{~kg}^{-1}$.
Carvalho (2000) verificou em relação ao nitrogênio para mucuna-preta, guandu, milheto e crotalária, no primeiro ano de cultivo, valores semelhantes aos do presente estudo (Tabela 2), corroborando que dentre esses adubos verdes a mucuna-preta apresentou maiores valores $(29,4$ $\left.\mathrm{g} \mathrm{kg}^{-1}\right)$. Em relação ao teor de fósforo (Tabela 2), Carvalho (2000) relata valores superiores aos deste estudo.

Não houve diferenças significativas entre as culturas de cobertura quanto ao teor de $\mathrm{K}$, apenas a área em pousio, onde se obteve o maior valor, diferindo significativamente dos outros tratamentos. De acordo com Teodoro et al. (2011) a alta capacidade de acúmulo de K apresentada pelas leguminosas torna-as uma boa alternativa para incremento desse elemento em sistemas nos quais sejam cultivadas principalmente espécies exigentes nesse nutriente - como é o caso das espécies acumuladoras de amido em partes subterrâneas.

Tabela 1. Matéria fresca e seca das plantas de cobertura aos 120 dias após a semeadura, em cultivos isolado e consorciados

\begin{tabular}{|c|c|c|}
\hline \multirow{2}{*}{$\begin{array}{l}\text { Culturas } \\
\text { de cobertura }\end{array}$} & Matéria fresca & Matéria seca \\
\hline & \multicolumn{2}{|c|}{$\left(\mathrm{t} \mathrm{h} \mathrm{a}^{-1}\right)$} \\
\hline Guandu & $25,6 \mathrm{c}$ & $7,7 \mathrm{C}$ \\
\hline Crotalária & $34,6 \mathrm{c}$ & $12,0 \mathrm{~B}$ \\
\hline Feijão-de-porco & 29,4 c & $6,0 \mathrm{C}$ \\
\hline Mucuna & $17,1 \mathrm{c}$ & 3,3 C \\
\hline Milheto & $42,7 \mathrm{~b}$ & $12,6 \mathrm{~B}$ \\
\hline Guandu + milheto & $44,1 \mathrm{~b}$ & $12,1 \mathrm{~B}$ \\
\hline Crotalária + milheto & $50,6 \mathrm{~b}$ & $14,8 \mathrm{~B}$ \\
\hline Feijão-de-porco + milheto & $47,4 \mathrm{~b}$ & $13,2 \mathrm{~B}$ \\
\hline Mucuna + milheto & $81,5 \mathrm{a}$ & $23,3 \mathrm{~A}$ \\
\hline Pousio & $31,2 \mathrm{c}$ & $6,7 \mathrm{C}$ \\
\hline $\mathrm{CV}(\%)$ & 33,7 & 36,8 \\
\hline
\end{tabular}

* Médias seguidas pela mesma letra na coluna não diferem entre si, pelo teste Scott-Knott, a 5\% de probabilidade. 
O maior teor de Ca foi encontrado no feijão-de-porco, que diferiu dos outros tratamentos, seguido da mucuna, crotalária, dos consórcios, do guandu e, por último, do milheto. Teodoro et al . (2011) obtiveram teor de Ca superior para o feijão-de-porco e crotalária juncea em relação às demais leguminosas testadas

Os teores de magnésio e enxofre foram similares aos relatados por (Tabela 2), Carvalho (2000) sendo importante ressaltar que o experimento foi realizado próximo ao local do experimento do presente trabalho.

Apenas para o teor de boro (Tabela 2) os valores foram superiores, em cultivo isolado, quando comparado com o consorciado; para os demais micronutrientes, o consórcio resultou em teores maiores do que o cultivo isolado, tendo a área em pousio apresentado teores elevados de micronutrientes.

Considerando-se o acúmulo de nutrientes proporcionado pelas diferentes combinações de tratamentos os agricultores podem optar pelas espécies mais adequadas, uma vez que pelos dados verifica-se que ocorreu acúmulo diferencial de nutrientes em razão da cultura isolada ou do tipo de consórcio utilizado com o milheto.

O uso dos adubos verdes em consórcio torna-se bastante interessante pelo acúmulo de nutrientes (Tabela 3). Pela elevada quantidade de matéria seca produzida, a combinação mucuna + milheto proporcionou a obtenção de elevada ciclagem de macro e micronutrientes, podendo fornecer às culturas sucessoras nutrientes em quantidade favorecendo o seu desenvolvimento e proporcionando incrementos em produtividade.

Oliveira et al. (2002), trabalhando com plantas de cobertura, dentre elas o milheto, a mucuna, o feijão-de-porco e o consórcio das leguminosas com o milheto, obtiveram valores de acúmulo de nutrientes inferiores aos do presente trabalho para quase todos os nutrientes, exceto para o potássio no feijão-de-porco $\left(50 \mathrm{~kg} \mathrm{ha}^{-1}\right)$, milheto (258 kg ha-1), enxofre no milheto (26 kg ha-1) e Mn para o milheto (1409 $\left.\mathrm{g} \mathrm{ha}^{-1}\right)$.

Tabela 2. Teores de nutrientes na parte aérea das diferentes plantas de cobertura do solo cultivadas isoladamente e em consórcio aos 120 dias após o plantio

\begin{tabular}{|c|c|c|c|c|c|c|c|c|c|c|}
\hline \multirow{2}{*}{$\begin{array}{l}\text { Plantas } \\
\text { de cobertura }\end{array}$} & \multicolumn{5}{|c|}{ Macronutrientes $\left(\mathrm{g} \mathrm{kg}^{-1}\right)$} & \multicolumn{5}{|c|}{ Micronutrientes (mg kg-1) } \\
\hline & $\mathbf{N}$ & $\mathbf{P}$ & $\mathbf{K}$ & $\mathbf{C a}$ & Mg & S & B & Fe & Mn & Zn \\
\hline Guandu & $21,9 \mathrm{c}$ & $2,7 \mathrm{~b}$ & $8,4 \mathrm{~b}$ & $9,3 \mathrm{c}$ & $2,5 \mathrm{c}$ & $1,4 \mathrm{a}$ & $40,1 \mathrm{~b}$ & $471,1 \mathrm{~b}$ & $46,1 \mathrm{c}$ & $21,4 \mathrm{~b}$ \\
\hline Crotalária & $16,4 \mathrm{~d}$ & $2,0 \mathrm{~d}$ & $7,3 \mathrm{~b}$ & $11,1 \mathrm{~b}$ & $4,1 \mathrm{a}$ & $1,4 \mathrm{a}$ & $41,0 \mathrm{~b}$ & $581,6 \mathrm{~b}$ & $55,1 \mathrm{c}$ & $17,4 \mathrm{~b}$ \\
\hline Feijão-de-porco & $31,0 \mathrm{~b}$ & 3,0 a & $8,0 \mathrm{~b}$ & $21,7 \mathrm{a}$ & $3,6 \mathrm{~b}$ & $1,6 \mathrm{~b}$ & $49,8 \mathrm{a}$ & 814,4 a & $63,0 \mathrm{~b}$ & $20,4 \mathrm{~b}$ \\
\hline Mucuna & $35,1 \mathrm{a}$ & $2,5 b$ & $8,4 \mathrm{~b}$ & $12,1 \mathrm{~b}$ & $3,0 \mathrm{c}$ & $1,7 \mathrm{~b}$ & $41,1 \mathrm{~b}$ & 773,3 a & $74,8 \mathrm{~b}$ & $19,0 \mathrm{~b}$ \\
\hline Milheto (M) & $13,3 \mathrm{~d}$ & $2,0 \mathrm{~d}$ & $6,9 \mathrm{~b}$ & $6,0 \mathrm{~d}$ & $4,5 \mathrm{a}$ & $1,2 \mathrm{a}$ & $32,8 \mathrm{c}$ & $560,5 \mathrm{~b}$ & $73,5 \mathrm{~b}$ & 26,0 a \\
\hline Guandu+(M) & $15,6 \mathrm{~d}$ & $1,9 \mathrm{~d}$ & $8,5 \mathrm{~b}$ & $7,6 \mathrm{c}$ & $3,8 \mathrm{~b}$ & $1,2 \mathrm{a}$ & $38,5 \mathrm{~b}$ & $616,3 \mathrm{~b}$ & $71,9 \mathrm{~b}$ & $23,8 \mathrm{a}$ \\
\hline Crotalária+(M) & $15,4 \mathrm{~d}$ & $1,9 \mathrm{~d}$ & $7,0 \mathrm{~b}$ & $8,9 \mathrm{c}$ & $4,7 \mathrm{a}$ & $1,4 \mathrm{a}$ & $36,3 \mathrm{~b}$ & $623,3 \mathrm{~b}$ & $71,4 \mathrm{~b}$ & $19,3 \mathrm{~b}$ \\
\hline Feijão-de-porco+(M) & $16,1 \mathrm{~d}$ & $2,0 \mathrm{~d}$ & $7,3 \mathrm{~b}$ & $7,8 \mathrm{c}$ & 4,8 a & $1,4 \mathrm{a}$ & $26,4 \mathrm{c}$ & $464,5 \mathrm{~b}$ & 86,8 a & $25,1 \mathrm{a}$ \\
\hline Mucuna+(M) & 20,3 c & $2,2 \mathrm{~d}$ & $8,2 \mathrm{~b}$ & $8,5 \mathrm{c}$ & $5,2 \mathrm{a}$ & 1,5 a & $29,8 \mathrm{c}$ & $515,0 \mathrm{~b}$ & $94,2 \mathrm{a}$ & $27,8 \mathrm{a}$ \\
\hline Pousio & $17,4 \mathrm{~d}$ & $2,3 c$ & $11,2 \mathrm{a}$ & $8,7 \mathrm{c}$ & 4,9 a & $2,0 \mathrm{~b}$ & $32,0 \mathrm{c}$ & 888,1 a & $96,4 \mathrm{a}$ & $17,8 \mathrm{~b}$ \\
\hline$\overline{\mathrm{CV}(\%)}$ & 14,8 & 14,1 & 25,5 & 19,4 & 17,0 & 20,8 & 24,1 & 40,6 & 19,8 & 24,7 \\
\hline
\end{tabular}

* Médias seguidas pela mesma letra nas colunas não diferem entre si, pelo teste Scott-Knott, a 5\% de probabilidade.

Tabela 3. Acúmulo de nutrientes da parte aérea das plantas de cobertura do solo cultivadas isoladamente e em consórcio aos 120 dias após o plantio

\begin{tabular}{|c|c|c|c|c|c|c|c|c|c|c|}
\hline \multirow{2}{*}{$\begin{array}{l}\text { Plantas } \\
\text { de cobertura }\end{array}$} & \multicolumn{6}{|c|}{ Macronutrientes (kg ha $\left.{ }^{-1}\right)$} & \multicolumn{4}{|c|}{ Micronutrientes (g ha ${ }^{-1}$ ) } \\
\hline & $\mathbf{N}$ & $\mathbf{P}$ & $\mathbf{K}$ & Ca & Mg & S & B & $\mathbf{F e}$ & Mn & $\mathrm{Zn}$ \\
\hline Guandu & $168 \mathrm{~b}$ & $20 \mathrm{~b}$ & $66 \mathrm{c}$ & $73 \mathrm{c}$ & $20 c$ & $10 \mathrm{c}$ & $321 \mathrm{~b}$ & $3558 \mathrm{~b}$ & $366 c$ & $156 c$ \\
\hline Crotalária & $201 \mathrm{~b}$ & $24 \mathrm{~b}$ & $87 \mathrm{~b}$ & $136 \mathrm{~b}$ & $50 \mathrm{~b}$ & $17 \mathrm{~b}$ & 516 a & $7044 \mathrm{~b}$ & $678 \mathrm{~b}$ & $207 \mathrm{~b}$ \\
\hline Feijão-de-porco & $186 \mathrm{~b}$ & $18 \mathrm{c}$ & $50 \mathrm{c}$ & $131 \mathrm{~b}$ & $22 \mathrm{c}$ & $10 \mathrm{c}$ & $288 \mathrm{~b}$ & $4954 \mathrm{~b}$ & $377 c$ & $125 \mathrm{c}$ \\
\hline Mucuna & $117 \mathrm{~b}$ & $8 \mathrm{c}$ & $29 c$ & $41 \mathrm{c}$ & $10 \mathrm{c}$ & $6 c$ & $137 \mathrm{~b}$ & $2569 \mathrm{~b}$ & $250 \mathrm{c}$ & $63 c$ \\
\hline Milheto (M) & $170 \mathrm{~b}$ & $28 \mathrm{~b}$ & $87 \mathrm{~b}$ & $76 \mathrm{c}$ & $58 \mathrm{~b}$ & $15 b$ & $415 \mathrm{a}$ & $6616 b$ & $944 \mathrm{~b}$ & $316 b$ \\
\hline Guandu+(M) & $186 \mathrm{~b}$ & $23 \mathrm{~b}$ & $101 \mathrm{~b}$ & $93 \mathrm{c}$ & $45 \mathrm{~b}$ & $15 b$ & $474 \mathrm{a}$ & $6969 \mathrm{~b}$ & $812 b$ & $273 b$ \\
\hline Crotalária+(M) & $226 \mathrm{~b}$ & $27 \mathrm{~b}$ & $102 \mathrm{~b}$ & $128 \mathrm{~b}$ & $68 \mathrm{~b}$ & $20 \mathrm{~b}$ & $530 \mathrm{a}$ & 9398 a & $1040 \mathrm{~b}$ & $280 \mathrm{~b}$ \\
\hline Feijão-de-porco+(M) & $212 b$ & $25 \mathrm{~b}$ & $95 \mathrm{~b}$ & $104 \mathrm{c}$ & $64 \mathrm{~b}$ & $18 \mathrm{~b}$ & $349 \mathrm{~b}$ & $6751 \mathrm{~b}$ & $1148 \mathrm{~b}$ & $323 \mathrm{~b}$ \\
\hline Mucuna+(M) & $473 \mathrm{a}$ & $52 \mathrm{a}$ & $195 \mathrm{a}$ & $201 \mathrm{a}$ & $126 \mathrm{a}$ & $34 \mathrm{a}$ & $682 a$ & 12229 a & 2237 a & $646 a$ \\
\hline Pousio & $115 \mathrm{~b}$ & $15 \mathrm{c}$ & $69 c$ & $56 \mathrm{c}$ & $30 \mathrm{c}$ & $11 \mathrm{c}$ & $220 \mathrm{~b}$ & $5721 b$ & $629 c$ & $112 \mathrm{c}$ \\
\hline$\overline{\mathrm{CV}(\%)}$ & 40,1 & 38,8 & 44,7 & 45,4 & 47,3 & 20,8 & 46,1 & 55,1 & 45,5 & 43,3 \\
\hline
\end{tabular}

* Médias seguidas pela mesma letra na coluna não diferem entre si, pelo teste Scott-Knott, a 5\% de probabilidade. 
Sendo assim, a utilização de adubo verde pode trazer benefícios pelo acúmulo de nutrientes presentes na matéria seca dessas plantas, proporcionando melhoria do solo e economia em termos de adubação.

\section{CONCLUSÃO}

Todos os consórcios que incluíram milheto apresentaram os maiores acúmulos de matéria seca, assim como os cultivos de milheto e crotalária juncea solteiros.

O maior acúmulo de nitrogênio da parte aérea foi obtido com utilização de mucuna, guandu e feijão-de-porco e para fósforo foi obtido com utilização da mucuna e do feijão-de-porco.

As espécies e consórcios estudados não apresentaram diferença em relação ao acúmulo de potássio da parte aérea das plantas.

\section{AGRADECIMENTOS}

À FAPESP - Fundação de Amparo à Pesquisa do Estado de São Paulo, pelo apoio financeiro.

\section{REFERÊNCIAS}

Ae N Arihara K, Okada K, Yosihara T \& Johansen C (1990) Phosphorus uptake by pigeonpea and its role in cropping systems of Indian Subcontinent. Science, 248:477-480.

Alvarenga RC, Cabezas WAL, Cruz JC, Santana DP (2001) Plantas de cobertura de solo para sistema plantio direto. Informe Agropecuário, 22:25-36.

Amabile RF, Fancelli AL \& Carvalho AM (2000) Comportamento de espécies de adubos verdes em diferentes épocas de semeadura e espaçamentos na região dos Cerrados. Pesquisa Agropecuária Brasileira, 35:47-54.

Amabile RF (1993) Coleção de espécies vegetais para cobertura e conservação dos solos sob vegetação de Cerrado: projeto de pesquisa. Planaltina, Embrapa-CPAC. 4p.

Ambrosano EJ \& Wutke EB (1997) Leguminosas adubos verdes Crotalária, chícaro ou ervilhaca, feijão-de-porco, feijão-guandu, lablabe, mucuna, tremoço. In: Raij BV, Cantarella H, Quaggio JA \& Furlani AMC (Eds.) Recomendações de adubação e calagem para o Estado de São Paulo. 2.ed. Campinas, IAC. 200p. (boletim técnico, 100).

Banzatto DA \& Kronka SN (2006) Experimentação agrícola. 4 ed. Jaboticabal, FUNEP. 237 p.

Bertolin DC, Sá ME, Buzetti S, Colombo AS, Oliveira LL \& Teixeira Filho MCM (2008) Doses de fósforo, potássio e espaçamentos entre linhas na produção de sementes e matéria de guandu em semeadura tardia. Scientia Agraria, 9:261-268.

Bordin I, Neves CSVJ, Francio Filho P, Preti EA \& Cardoso C (2008) Crescimento de milheto e guandu, desempenho de plantas cítricas e propriedades físicas do solo escarificado em um pomar. Revista Brasileira de Ciência do Solo, 32:14091418.

Braz AJBP, Kliemann HJ \& Silveira PM (2010) Produtividade de palhada de plantas de cobertura. In: Silveira PM \& Stone LF Plantas de cobertura dos solos do Cerrado. p.13-43. (Eds.)
Carvalho MAC (2000) Adubação verde e sucessão de culturas em semeadura direta e convencional em Selvíria-MS. Tese de Doutorado. Universidade Estadual Paulista “Júlio de Mesquita Filho", Jaboticabal. 189p.

Embrapa. Empresa Brasileira De Pesquisa Agropecuária (1999) Sistema brasileiro de classificação de solos. Rio de Janeiro, EMBRAPA/CNPSO. 412p.

Ferrari Neto J, Crusciol CAC, Soratto RP, Costa CHM da (2011) Plantas de cobertura, manejo da palhada e produtividade da mamoneira no sistema plantio direto. Revista Ciência Agronômica, 42:978-985.

Heinrichs R, Vitti GC, Moreira A, Figueiredo AM, Fancelli AL \& Corazza EJ (2005) Características químicas de solo e rendimento de fitomatéria de adubos verdes e de grãos de milho decorrente do cultivo consorciado. Revista Brasileira de Ciência do Solo, 29:71-79.

Oliveira AS (2002) Produção de forragem e de sementes de Brachiaria decumbens Stapf em função da adubação com nitrogênio e fósforo e cultura antecessora. Dissertação de Mestrado. Universidade Estadual Paulista “Júlio de Mesquita Filho”, Ilha Solteira. 87p.

Oliveira TK, Carvalho GJ \& Moraes RNS (2002) Plantas de cobertura e seus efeitos sobre o feijoeiro em plantio direto. Pesquisa Agropecuária Brasileira, 37:1079-1087.

Pereira J (1991) Avaliação das características agronômicas de leguminosas adubos verdes nos Cerrado. Relatório Técnico Anual do Centro de Pesquisa Agropecuária do Cerrado 1985/1987, Planaltina. p.111-112.

Pitol C, Broch LD, Carvalho AM \& Spera TS (2006) Uso de adubos verdes nos sistemas de produção no Bioma Cerrado. In: Carvalho AM \& Ambile RF (Eds.) Cerrado: adubação verde. Planaltina, Embrapa Cerrados. p.301-330.

Raij BV \& Quaggio JA (1983) Métodos de análise de solo para fins de fertilidade, Campinas. 31p. (Boletim Técnico, 81).

Salton JG, Pitol C \& Erbes E (1993) Cultivo de primavera: alternativa para produção de palha em Mato Grosso do Sul. Maracaju, Fundação MS. 6p. (Informativo Técnico, 1).

Silva PCG, Foloni JSS, Fabris LB \& Tiritan CS (2009) Fitomassa e relação $\mathrm{C} / \mathrm{N}$ em consórcios de sorgo e milho com espécies de cobertura. Pesquisa Agropecuária Brasileira, 44:1504-1512.

Spehar CR (1999) Sistemas de produção de milheto nos Cerrados. In: Workshop Internacional de Milheto, Planaltina. Anais Embrapa Cerrados. p.69-73.

Teodoro RB, Oliveira FL de, Silva DMN da, Fávero C, Quaresma MAL (2011) Aspectos agronômicos de leguminosas para adubação verde no Cerrado no Alto Vale do Jequitinhonha. Revista Brasileira de Ciência do Solo, 35:635-643.

Zonta EP \& Machado AA (1991) SANEST-Sistema de análise de variância para microcomputadores. Pelotas, UFPel.104p. 